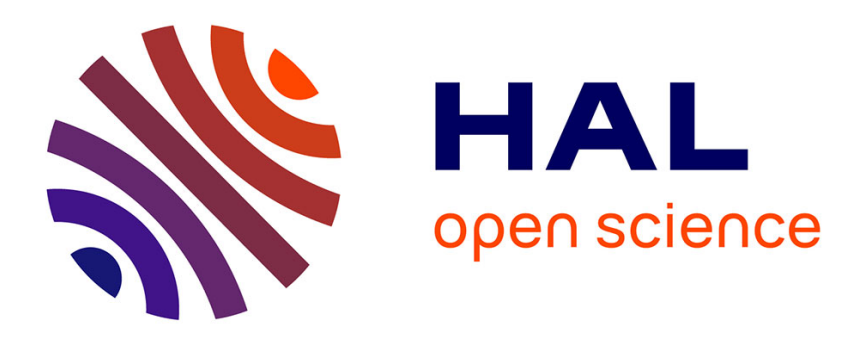

\title{
Non-Linear Magneto-Optic - Microwave Interactions : a New Range of Devices
}

\author{
S. Nikitov
}

\section{To cite this version:}

S. Nikitov. Non-Linear Magneto-Optic - Microwave Interactions : a New Range of Devices. Journal de Physique IV Proceedings, 1997, 07 (C1), pp.C1-703-C1-706. 10.1051/jp4:19971286 . jpa-00254999

\section{HAL Id: jpa-00254999 https://hal.science/jpa-00254999}

Submitted on 1 Jan 1997

HAL is a multi-disciplinary open access archive for the deposit and dissemination of scientific research documents, whether they are published or not. The documents may come from teaching and research institutions in France or abroad, or from public or private research centers.
L'archive ouverte pluridisciplinaire HAL, est destinée au dépôt et à la diffusion de documents scientifiques de niveau recherche, publiés ou non, émanant des établissements d'enseignement et de recherche français ou étrangers, des laboratoires publics ou privés. 


\title{
Non-Linear Magneto-Optic - Microwave Interactions: a New Range of Devices
}

\author{
S.A. Nikitov \\ Institute of Radioengineering \& Electronics, Russian Academy of Sciences, 11 Mokhovaya St., 103907 , \\ Moscow, Russia
}

\begin{abstract}
Ferrite films of yttrium-iron-garnet (YTG) are perfect candidates for various applications in microwave and infrared frequency range. The theoretical and experimental results of intense study of interaction of spin waves with light in such films are reviewed and practical applications are presented. Along with traditional methods of magneto-optic - spin wave interaction the novel methods including non-linear waves are considered. Traditional devices include frequency shifters, scanners, modulators, but a new class of non-linear devices is suggested and practical tecommendations are provided.
\end{abstract}

\section{INTRODUCTION}

Ferrite films of YIG have currently found various applications. These films grown usually by the liquid-phase epitaxy method possess a good quality suitable for both microwave and optic waves applications. First of all, these films with the thickness in few (or tens) of microns and lateral dimensions in one or even few centimeters possess properties of ferromagnetic medium at the frequency range from 1 to $20 \mathrm{GHz}$. This frequency range is intensively studied due to possibility to excite and investigate traveling magnetostatic, spin waves (MSW). On the other hand, in the optical infrared wavelength range between 1.1 and 6 $\mu m$ YJG films find an application due to strong efficiency of magneto-optic interactions. Light propagates in such films as waveguided modes and they become coupled and can be converted due to Faraday and Cotton-Mouton magneto-optic effect. Usually YIG films sitting on gadolinium-gallium-garnet (GGG) substrates are used for the purposes of the strong optical mode conversion and this finds different appiications. Yet, in order to increase optical mode conversion Bi- and Ce-doped YIG films are the best sources. The most interesting applications, however, can be achieved when both microwave and light waves propagate simultaneously in such films. The possibility of such a film to support the waves is used for effective magneto-optic Bragg interaction between these vaves [1,2]. Frequencies and wavenumbers of MSW are usually much smaller than the respective values for optic waves. Thus under the special relation between the wavenumbers of the incident and diffracted light modes and the MSW wavenumber, the anisotropic Bragg diffraction of light can be quite essential. In following; the basic methods of magneto-optic - microwave interactions will be described and new results and novel applications will be provided.

\section{ANISOTROPIC BRAGG DIFFRACTION}

Light propagating in a magnetic film is represented as a set of TE and TM modes. Due to magneto-optic effects they become coupled. The propagating MSW creates a dynamic phase diffraction lattice for light in a ferrite film. Since the wavenumbers of the coupled modes differ, essential diffraction (with mode conversion) can be achieved only when the phase-matching condition is fulfilled. Based on the coupled-mode theory, the following requirement should be fulfilled in order to obtain maximum mode conversion

$$
\Delta=k_{T E}-k_{T M}-Q_{M S W},
$$

where $k_{T E}, k_{T M}$ are the optical modes' wave vectors and $Q_{M S W}$ is the MSW wave vector. The conversion efficiency between the modes during propagation is

$$
\eta^{ \pm}=\sin ^{2}\left(\delta^{ \pm} L\right)
$$

where $\mathrm{L}$ is the propagation distance and $\delta^{*}$ is the mode conversion coefficient for TE or TM modes. In particuiar, it is proportional to the MSW power. Therefore. in order to achieve strong optical conversion the MSW power should be quite high. In good quality Bi- substituted YIG films, the conversion efficiency can be about $40 \%$ at the MSW power level of abont

1 W per unit width. However, at this power level the MSW become strongly non-linear and this, in turn, causes various 
undesirable effects. Among them appearance of new waves in the MSW spectrum; saturation of the MSW power; non-linear interaction between MSW and light, etc, most often can be met in an experiment. Since optical mode conversion is a linear effect and is supposed to be used in linear devices, all these non-linear effects accompanying traveling intensive MSW can be separated and used for novel, yet, important applications. Some of them will be presented below.

\section{NON-LINEAR INTERACTIONS BETWEEN MSW AND LIGHT}

\subsection{Bistability in optical mode conversion}

Optical mode conversion in magnetic waveguides appears due to tensorial dependence of the dielectric permittivity upon the magnetic fields (magnetization) in the MSW. In case of non-linear MSW this tensor is

$$
\varepsilon_{i j}=\alpha_{i j k} M_{k}+\beta_{i j k i} M_{k} M_{l}
$$

where $\alpha_{i j k}, \beta_{i j k l}$ are the magneto-optic constants; $M_{i}(i=x, y, z)$ are the magnetization components and the non-linear MSW terms appear through the expression $M_{z} \cong M_{0}\left(1-\left(M_{x}^{2}+M_{y}^{2}\right) / 2 M_{0}^{2}\right)$, where $M_{0}$ is the saturation magnetization. Coupled-wave theory gives the value for the coupling parameter $\delta^{ \pm}[3]$ :

$$
\delta^{ \pm}=\frac{\gamma_{1}+\gamma_{2}-\Delta}{2} \pm\left(F+\frac{\left(\gamma_{1}+\gamma_{2}\right)^{2}}{4}+\gamma_{1} \gamma_{2}+\frac{\Delta}{4}\left(\Delta+\frac{\gamma_{1}-\gamma_{2}}{2}\right)\right)^{\frac{1}{2}}
$$

where $\gamma_{1.2}$ are pure imaginary and $F, \gamma_{1.2}$ depend on the MSW power and magneto-optic constants. In the linear case $\gamma_{1,2}=0$, and $\delta^{ \pm}$are pure real. The non-linearity makes $\delta^{ \pm}$complex values and imposes strong asymmetry to the dependence $\delta^{ \pm}(\omega)$ on $\Delta \omega$, where $\Delta \omega$ is a deviation of the light frequency about the point corresponding maximum conversion. Bistability is a result of the non-linearity imposed MSW frequency shift. At high level of the microwave power the MSW frequency is shifted, although the excitation microwave frequency is not. This shift breaks down the phase-matching condition (1) and, consequently, changes the conversion efficiency. Thus this efficiency is not the same at different power levels and, moreover, bistability has a power threshold. The estimated power threshold is less than the parametric instability power threshold. Therefore, this effect can be easily recognized in microwave -- magneto-optic experiment. The dependence of the conversion efficiency on the input MSW power makes it possible to control the phase-matching condition with the microwave power.

\subsection{Fibre-optic frequency shifter using non-linear MSW}

Intensive non-linear MSW can be unstable to various parametric and other non-linear processes. Parametric instability leads to appearance of the waves with different frequencies as a result of the parametric decay. These waves destroy the optical mode coupling and conversion and, thereby, the instability of MSW should be avoided during MSW -- magneto-optic interaction. It is possible to distinguish the different parametric processes by proper choice of the microwave frequency and other experimental parameters. Even if the low power threshold parametric instability of $c w$ MSW is avoided, the wave still can be subjected to longitudinal or transverse instability. In the first case, it is modulation instability; in the second case, it is self-focusing of the MSW beam. The latter effect can be quite important in magneto-optic -- microwave applications.

Let assume the MSW beam can be focused into a narrow focal spot by specially prepared excitation antenna and/or in the case, when non-linearity induces the beam focusing. Perpendicularly to the MSW wavefront a piece of an optical fibre is put and pressed against the film surface. Assume further that the fibre is the two-mode and the only spatial modes $L P_{01}$ and $L P_{11}$ can propagate in it. The MSW beam with the initial aperture of about $1.4 \mathrm{~mm}$ can be focused into the spot of a diameter of about $0.6 \mathrm{~mm}$ at the propagation distance $5 \mathrm{~mm}$. The peak intensity of the magnetization (and, respectively, magnetic field) is rather high in the focusing area. The light entering a fibre consists of only the $L P_{01}$ mode. MSW in the focal spot generates the spatial dynamic grating of the electromagnetic field. The frequency of this field is equal to the frequency difference between $L P_{01}$ and $L P_{11}$ modes. This field couples the two spatial fibre modes. Thus the conversion between the modes occurs. The conversion efficiency is proportional to the MSW power and the coupling between the electromagnetic field of the MSW and the optic modes. Optimum mode-coupling efficiency was found to be for the MSW with the frequency $f=1.22$ $\mathrm{GH}$, and the beat-length distance was about $200 \mu \mathrm{m}$. For MSW with the power $100 \mathrm{~mW}$ the mode-coupling efficiency is $10^{-3}$ (or $0.1 \%$ ). The efficiency can be increased by using higher microwave power and improving the coupling between the film and fibre. This method of the mode coupling can be used in diagnostics and production control [4]. 


\subsection{Optic mode compression}

Consider now the propagation of the narrow optic pulse in a ferrite film with the chirped MSW. The MSW induces the phase diffraction lattice with the linearly variable period. Therefore the different spectral components of the pulse possess the diffraction at the different segments of the film. If $\omega$ and $c$ are the light frequency and velocity; $\Omega$ and $v$ are the MSW frequency and velocity, the synchronism between the instantaneous light frequency and the MSW frequency is fulfilled through the following equation

$$
\Omega=\omega \frac{v \Delta n}{c},
$$

where $\Delta n$ is an optical indices difference for incident and diffracted waves. The efficiency of diffraction is enhanced for the case when the group velocity of the diffracted light is equal to the slope of the synchronism line defined by equation (5). Therefore the rate of the frequencies variation is followed from the relation:

$$
\frac{d \Omega}{d t}=\frac{d \omega}{d t} *\left(\frac{v \Delta n}{c}\right)^{2}
$$

If the amplitude of the incident light is $A(\tau)$ then the output field is

$$
A_{\text {out }}(t)=\varepsilon \int_{t-\frac{t_{0}}{2}}^{t+\frac{t_{0}}{2}} A(\tau) \exp [i \varphi(\tau)-i \omega \tau] d \tau,
$$

where $\varepsilon$ is a coupling coefficient between optic modes that is proportional to the spin wave amplitude; $t_{0}=L(\Delta n / c)$ is the delay time; $L$ is the crystal length and $\varphi(\tau)$ is a phase shift imposed by the group-velocity dispersion. If the initial pulse is of the squared shape the maximum output amplitude is

$$
A_{\max } \approx A_{0} \varepsilon L_{D}
$$

and $L_{D}$ is the effective dispersion length. This is the distance over which the pulse doubles its width due to induced dispersion. It is defined as

$$
L_{D}=\frac{c T_{0}}{\Delta n}
$$

and $T_{0}$ is the initial pulse width. The least width of the compressed pulse is

$$
T_{\min }=\frac{a}{\left(\frac{d \omega}{d t}\right) T_{0}}
$$

and $a$ is a numerical coefficient approximately equal to 3. The typical YIG films used in experiments with MSW are shorter than $1 \mathrm{~cm}$ and this restriction are due to the MSW propagation losses. Thus the dispersion length of the order of few millimeters can be achieved for the optical pulses in the picosecond range (or shorter). Compressing efficiency for such a length of a crystal can be more than 10 for picosecond pulses. To obtain a shorter compressed pulse one should repeat the compressing procedure several times using the same YIG film.

\subsection{Spin wave pulse generation}

In this Section it will be shown how the spin wave pulse can be generated under the mutual interaction between two orthogonally polarized optical pulses (or between two components of the same intensive ultrashort pulse) [5]. TE and TM light modes guided by the ferrite film have different dispersive characteristics and even for the same frequency propagate with different velocities. Assume now that the two ultrashort optical pulses with different polarization are excited in the same ferrite film. The pulse having smaller velocity is the first launched into the film. The second pulse launched with some delay will keep ahead the first pulse if its velocity is greater the velocity of the first pulse. In the region of overlapping of two pulses the dynamic phase grating of the electromagnetic field will occur. This grating will be spatially periodic. The wavenumber of this grating is equal to the wavenumbers' difference of the respective modes

$$
Q=K_{T E}-K_{T M}
$$

The dimensions of this grating in time and space are defined by the following expressions:

$$
\begin{aligned}
& t=T * \frac{\left(K_{T E}+K_{T M}\right)}{\left(K_{T E}-K_{T M}\right)} \\
& l=v_{T E} t
\end{aligned}
$$

where $T$ is a pulse duration and $v_{T E}$ is the velocity of the light respecting to the TE mode. In the region of the film with periodic variations of the electromagnetic field two spin wave pulses propagating in opposite directions will be excited if the coupling efficiency is sufficient. The frequency of these spin waves is expressed as

$$
\Omega=\omega * \frac{v \Delta n}{c},
$$


where $W$ is the frequency of the light, $\Delta n$ is the difference between the optic indices for TE and TM modes and $v$ is a MSW group velocity. The duration of the MSW pulse is

$$
T_{M S W}=T * \frac{v_{T E}}{v} * \frac{\left(K_{T E}+K_{T M}\right)}{\left(K_{T E}-K_{T M}\right)}
$$

The ratio $v_{T E} / v$ is usually of the order of $10^{3}-10^{4}$, thus making the value for $T_{\text {MSW }}$ greater than $T$ in about 3 orders of magnitude. Therefore, exciting the optical pulses in picosecond range of duration one can obtain the MSW pulses in a nanosecond scale.

To get the solution for the magnetization amplitude in the MSW one should consider the Landau-Lifshitz equation for the magnetization. Solving this equation by methods of the theory of perturbation, one obtains the expression for the RF magnetization:

$$
m_{x}=i m_{y}=-\frac{\gamma M_{0}}{4 \Omega \Delta H} * h_{z} h_{x}^{*} A\left(H_{0}, d\right),
$$

where $\gamma$ is a gyromagnetic ratio, $M_{0}$ is a saturation magnetization, $\Delta H$ is a ferromagnetic resonance line width, $h_{z}, h_{x}$ are the RF components of the amplitudes of the magnetic field in the light and $A\left(H_{0}, d\right)$ is a coefficient depending on an external magnetic field and the film thickness, * denotes the complex conjugate. Assuming the waves propagating along the $\mathrm{OY}$ axis and the magnetic field components of the following form

$$
\begin{aligned}
& h_{z}=A\left(t-\frac{y n_{T M}}{c}\right) * \cos \left[\omega\left(t-\frac{y n_{T M}}{c}\right)\right] \\
& h_{x}=A\left(t-t_{0}-\frac{y n_{T E}}{c}\right) * \cos \left[\omega\left(t-t_{0}-\frac{y n_{T E}}{c}\right)\right]
\end{aligned}
$$

where $A$ is the pulses envelope and $n_{T M}, n_{T E}$ are the optic indices for TM and TE modes, the expression for the RF nagnetization is:

$$
m_{y}=\frac{\gamma M_{0}}{4 \Omega \Delta H} * \cos \left(t-\frac{L}{v}\right) * F(t, L),
$$

$L$ is a crystal length and $F(\tau, L)=\int A(t) A(t-\tau) d t$ is a correlation function of the magnetization. Thus the magnetization envelope is proportional to the self-correlation function of the optical pulse. Estimating the efficiency of this magnetodynamic resonance one gets that the optical pulse with duration 1 ps and the intensity $10 \mathrm{MW} / \mathrm{cm}(\lambda \propto 1.3 \mu \mathrm{m})$ generate the MSW pulses with duration $3 \mathrm{~ns}$ and the peak power of about $20 \mu \mathrm{W}$. The pulses with such a power can be easily registrated in a microwave experiment.

\section{CONCLUSION}

Non-linear effects accompanying MSW - magneto-optic interaction whether they are pure microwave or magneto-optic origin can be used to build a new range of microwave and optic devices. The simple but important are modulators and digital matrix-vector multipliers working as digital processors. Effect of the light diffraction by the non-linear MSW provides the possibility to make the magneto-optic spatial Fourier-processor. It can realize the convolution of the two optical signals. Among the devices utilizing magneto-optic - MSW interaction are deflectors and nonreciprocal isolators, optical switchers and high-speed modulators. Non-linear properties of MSW are also used for generation and formation of spin wave solitons and shock waves. High-order solitons of MSW can be used for the efficient compression of MSW pulses. Compression efficiency is very high $(>50)$ at the distances of a few $\mathrm{mm}$. Spin wave bistability permits to develop nonreciprocal MSW devices. These devices along with optical devices can essentially extend the possibility of non-linear magneto-optics.

\section{Acknowledgments}

Financial support from the Russian Foundation for Basic Research, Grant 96-02-16168-a and from the Russian State Committee for the Science and Technology is gratefully acknowledged.

\section{References}

[1]. Tsai C.S. and Young D., IEEE Trans. on Microwave Theory and Tech. MTT-38 (1990) pp.560-570.

[2]. Stancil D.D., Theory of Magnetostatic Waves (Springer - Verlag, New York, 1993) pp.174-209.

[3]. Boardman A.D., Gulyaev Y.V. and Nikitov S.A., Jap. J. Appl. Phys. 27 (1988) pp.L2438-244].

[4]. Engan H.E., Myrtveit T. and Askautnd J.O., Opt. Lett. 16 (1991) pp.24-26.

[5]. Nikitov S.A., Gulyaev Y.V. and Pustovoit V.1. (to be published in Opt. Comm.). 